I N S T I T U T O

$\mathrm{DE}$

M E D I C I N A

T R O P I C A L

$\mathrm{DE}$

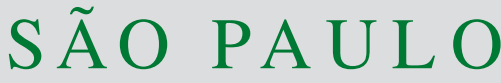

JOURNAL OF THE SÃO PAULO INSTITUTE OF TROPICAL MEDICINE

'Universidade Estadual de Campinas, Departamento de Pediatria, Campinas, São Paulo, Brazil

2Pontifícia Universidade Católica de Campinas, Campinas, São Paulo, Brazil

${ }^{3}$ Universidade Estadual de Campinas, Faculdade de Ciências Médicas, Laboratório de Pesquisa Aplicada em Dermatologia e Infecção por Bartonella, Campinas, São Paulo, Brazil

${ }^{4}$ Universidade Estadual de Campinas, Faculdade de Ciências Médicas, Departamento de Clínica Médica, Disciplina de Dermatologia, Campinas, São Paulo, Brazil

Correspondence to: Paulo Eduardo Neves Ferreira Velho

Universidade Estadual de Campinas, Faculdade de Ciências Médicas, Rua Tessália Vieira Camargo, 126, Barão Geraldo, CEP 13083-887, Campinas, SP, Brazil

Tel: + 5519 3521-9134

E-mail: pvelho@unicamp.br

Received: 26 August 2021

Accepted: 20 January 2022

\section{Two-year history of lymphadenopathy and fever caused by Bartonella henselae in a child}

\author{
Helga Kaiser Sanches de Maria', Emily Gazzoli², Marina Rovani Drummond ${ }^{1}$, \\ Amanda Roberta de Almeida $^{3}$, Luciene Silva dos Santos ${ }^{(103}$, Ricardo Mendes \\ Pereira' ${ }^{1}$, Antonia Terezinha Tresoldi', Paulo Eduardo Neves Ferreira Velho ${ }^{3,4}$
}

\section{ABSTRACT}

We report the case of a 6-year-old boy that presented with enlarged lymph nodes on his neck. He complained of tiredness and discouragement, which worsened during feverish periods. There were no relevant laboratory test abnormalities and serological tests were not reactive. Bartonella henselae DNA was detected by species-specific nested polymerase chain reaction. After treatment, the patient progressed with no fever or lymphadenopathy. Bartonellosis is a group of infectious diseases caused by bacteria of the genus Bartonella. This case report is a useful reminder to clinicians that long-term fever of unknown origin can be related to $B$. henselae infection, even if the specific serology is not reactive.

KEYWORDS: Bartonella. Diagnosis. Bartonellosis. Lymphadenopathy. Fever of unknown origin.

\section{CASE REPORT}

A 6-year-old boy was admitted to a Primary Health Care Unit (PHCU) in the

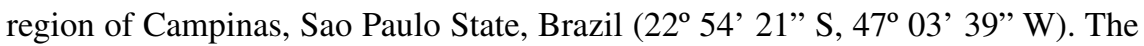
history of the present illness began two years earlier, when his mother noticed six nodes on his neck. By this time, they were living in Espirito Santo State (20 $19^{\prime} 48^{\prime \prime} \mathrm{S}, 40^{\circ} 17^{\prime} 31^{\prime \prime} \mathrm{W}$ ) and the cervical ultrasound examination showed lymph node images in the carotid chains and in the left posterior cervical jugular chains of non-specific etiology (the largest lymph node was $2.2 \times 1.6 \mathrm{~cm}$ ). The histopathological analyses of an excised lymph node showed chronic reactive lymphadenitis. Twenty days after the surgical procedure, new small lymph nodes appeared. Since then, the mother reports feverish periods, usually once a day, three peaks per week $\left(38-40.1^{\circ} \mathrm{C}\right)$. In the last 30 days she reported at least four peaks of $39.8,40.1,38.2$ and $40{ }^{\circ} \mathrm{C}$. The boy complained of tiredness and discouragement, that worsened during feverish periods. He referred eventual abdominal and limb pain, but continued to play with colleagues, with no restriction of activities and maintained good school performance. The mother also referred that the boy has contact with pets in the neighborhood, especially dogs and cats. There were no reports of travel, insect or animal bites, animal scratching, blood transfusions, and contact with sick people. The mother's gestational history was uneventful, and he was born by caesarean section. On physical examination, the patient was wellnourished. Tender, painless and mobile lymph nodes were palpable $(<1.0 \mathrm{~cm})$ in the posterior cervical, axillary and left inguinal chains. No palpable organomegaly was found. There were no relevant laboratory tests abnormalities including blood 
cell count, erythrocyte sedimentation rates, and serum protein electrophoresis.

Abdominal ultrasound and chest X-ray showed no abnormal findings. A positron emission tomography and computed tomography scans revealed multiple diffuse bilateral cervical lymph nodes (usually $1 \mathrm{~cm}$ on the shortest axis), rare sparse lymph node enlargement in the left posterior carotid chain and cervical jugular chains. Multiple axillary, mediastinal, retroperitoneal, iliac and inguinal lymph nodes were sparse, measuring less than $1 \mathrm{~cm}$, slightly enlargement liver and spleen, although still within the normal range for his age group. Serological testing for HIV, cytomegalovirus, Epstein-Barr virus, hepatitis A, B and C virus, Toxoplasma gondii, Trypanosoma cruzi, Histoplasma capsulatum, Coxiella burnetiid were not reactive. Using antigens supplied by the Centers for Disease Control and Prevention (CDC, Atlanta, USA), serum samples were analyzed for $\operatorname{IgG}$ antibodies to $B$. henselae and B. quintana antigens by an indirect immunofluorescence assay, as described previously ${ }^{1}$, and these serological tests were also not reactive. A tuberculin skin test was negative.

A blood sample was sent to the Applied Research in Dermatology and Bartonella Infection Laboratory, University of Campinas (UNICAMP) Medical School. Species-specific nested polymerase chain reaction (PCR) that amplifies Bartonella henselae fts $Z$ gene did not detect Bartonella DNA in a whole blood sample, but the amplification of an aliquot of a ten-day specific liquid culture detected $B$. henselae DNA. There were no Bartonella sp. isolates even when specific conditions of liquid and solid cultures were used. Primers and reagents conditions as well as of the amplifications have been already described, as well as the composition of the specific liquid and solid culture medium ${ }^{2,3}$.

The patient was referred to the UNICAMP hospital. Urine and blood cultures were negative. Anti-nucleus, anti-nucleolar, anti-cytoplasmatic, anti-nuclear mitotic apparatus, anti-metaphase chromosome factors were not reactive. The echocardiography was normal.

He was treated with intravenous gentamicin $(7.5 \mathrm{mg} / \mathrm{kg} / \mathrm{d})$ and ceftriaxone $(2 \mathrm{~g} / \mathrm{d})$ for two weeks and oral azithromycin $(500 \mathrm{mg} /$ day) for up to four months. At the end of this period, the patient presented with no fever or lymphadenopathy. After 36 months of outpatient follow-up, the patient showed no recurrence of signs and symptoms of bartonellosis.

\section{Ethical statement}

The child's mother signed a consent form authorizing the publication by the authors of her child's clinical case, including associated data and laboratory tests results.

\section{DISCUSSION}

Bartonellosis is a group of infectious diseases caused by bacteria of the genus Bartonella ${ }^{4}$. Bartonella henselae is among the most reported species related to human diseases. The main manifestation related to this species is the cat scratch disease (CSD) that is characterized by a 2-8 weeks self-limited lymphadenopathy that occurs mostly in children and does not require antibiotic therapy ${ }^{5}$.

We report the case of a boy that presented with enlarged lymph nodes on his neck. The cephalic region is the second most common location of lymphadenopathy in CSD (head and neck accounting for 26\%), after axillary and epitrochlear (27-46\%) ${ }^{6}$. This suggests that the $B$. henselae inoculation took place on the upper part of his body, even though there was no visible sign of animal scratch or flea bite. The common occurrence of patients with CSD makes this disease one of the most frequent causes of infectious cervical lymphadenopathy ${ }^{7}$.

The evolution of lymphadenopathy in CSD is usually subacute $^{5,6}$. When lymphadenopathy is persistent (more than 6 weeks) ${ }^{7}$ or malignancy is suspected, a biopsy should be considered $^{8}$. Our patient underwent a lymph node biopsy and the pathological analyses of an excised lymph node showed chronic reactive lymphadenitis. A few days after the surgical procedure, the patient began to have fever of unknown origin (FUO).

In a typical case of CSD, fever of short duration is a common finding with an average of six days ${ }^{9}$. However, this patient reported two years of fever, once a day, three peaks a week. A study carried out in Israel ${ }^{9}$ characterized CSD with FUO as a unique syndrome for which fever lasted a median of four weeks (range 2-9 days). The study concluded that prolonged FUO is more frequent in adults and may have a continuous or relapsing pattern, as well as multiorgan involvement.

Although some reports name other medical conditions such as chronic lymphadenopathy, FUO, erythema nodosum, ocular disease, encephalitis, osteomyelitis, hepatosplenic involvement as "atypical CSD" ${ }^{10}$, today these manifestations should be better described as bartonelloses, as many times they are not related to contact with cats or lymphadenopathy $y^{4,5,9,11-14}$.

Diagnosis of bartonellosis is a challenge considering the difficulty of culturing Bartonella spp.. Although serological assays are used to confirm Bartonella sp. infection, it is known that the sensitivity of this technique has been considered poor and that serological results lack correlation with bloodstream infection ${ }^{15,16}$. Even in the presence of a negative serology, PCR should be performed as it may detect $B$. henselae DNA in blood samples or biopsied 
tissues ${ }^{9,17}$. False negative results can happen even in the molecular diagnosis of bartonellosis ${ }^{14}$. The diagnosis can be facilitated if the assistant physician finds information about cat scratching or flea bites in the patient's history or a visible sign of animal aggression, however, the classical epidemiology of being a pet owner may lack ${ }^{18,19}$.

There are insufficient data to guide definitive recommendations for treating chronic $B$. henselae infections. A bactericidal antibiotic, such as an aminoglycoside, can be added to the treatment since macrolides have only bacteriostatic activity. In the case of Bartonella sp. endocarditis, a combined therapy should be adopted by the addition of azithromycin monotherapy for up to 6 months ${ }^{20}$. Considering the wide spectrum of clinical symptoms in bartonelloses and the difficulty of serological testing for $B$. henselae, the diagnosis depends on a combination of criteria. In this case, the patient's diagnosis was made based on a two-year history of lymphadenopathy and fever of unknown origin, exclusion of other diagnoses, positive PCR test for $B$. henselae DNA and recovery after treatment. This case report is a useful reminder to clinicians that long-term fever of unknown origin can be related to $B$. henselae infections even if the specific serology is not reactive.

\section{FUNDING}

Doctoral Scholarships from National Council for Scientific and Technological Development (CNPq) $\mathrm{N}^{\circ} 159717 / 2013-2$ (MRD) and 170501/2018-3 (LSS); Productivity Grant from CNPq No 306970/2018-0 (PENFV).

\section{REFERENCES}

1. Pitassi LH, Diniz PP, Scorpio DG, Drummond MR, Lania BG, Barjas-Castro ML, et al. Bartonella spp. bacteremia in blood donors from Campinas, Brazil. PLoS Negl Trop Dis. 2015;9:e0003467.

2. Drummond MR, Visentainer L, Almeida AR, Angerami RN, Aoki FH, Velho PE. Bartonella henselae bacteremia diagnosed postmortem in a myelodysplastic syndrome patient. Rev Inst Med Trop Sao Paulo. 2019;61:e50.

3. Drummond MR, Lania BG, Diniz PP, Gilioli R, Demolin DM, Scorpio DG, et al. Improvement of Bartonella henselae DNA detection in cat blood samples by combining molecular and culture methods. J Clin Microbiol. 2018;56:e01732-17.

4. Maguiña C, Guerra H, Ventosilla P. Bartonellosis. Clin Dermatol. 2009;27:271-80
5. Sandoval AC, Reyes FT, Prado MA, Peña AL, Viviani TN. Catscratch disease in the pediatric population: 6 years of evaluation and follow-up in a public hospital in Chile. Pediatr Infect Dis J. 2020;39:889-93.

6. Chang CC, Lee CJ, Ou LS, Wang CJ, Huang YC. Disseminated cat-scratch disease: case report and review of the literature. Paediatr Int Child Health. 2016;36:232-4.

7. Weinstock MS, Patel NA, Smith LP. Pediatric cervical lymphadenopathy. Pediatr Rev. 2018;39:433-43.

8. Nolder AR. Paediatric cervical lymphadenopathy: when to biopsy? Curr Opin Otolaryngol Head Neck Surg. 2013;21:567-70.

9. Landes M, Maor Y, Mercer D, Habot-Wilner Z, Bilavsky E, Chazan B, et al. Cat scratch disease presenting as fever of unknown origin is a unique clinical syndrome. Clin Infec Dis. 2020;71:2818-24

10. Nawrocki CC, Max RJ, Marzec NS, Nelson CA. Atypical manifestations of cat-scratch disease, United States, 20052014. Emerg Infect Dis. 2020;26:1438-46.

11. Lins KA, Drummond MR, Velho PE. Cutaneous manifestations of Bartonellosis. An Bras Dermatol. 2019;94:594-602.

12. Breitschwerdt EB. Bartonellosis: one health perspectives for an emerging infectious disease. ILAR J. 2014;55:46-58.

13. Hotez PJ, Aksoy S, Brindley PJ, Kamhawi S. What constitutes a neglected tropical disease? PLoS Negl Trop Dis. 2020;14: e0008001.

14. Drummond MR, Santos LS, Silva MN, Almeida AR, Diniz PP, Angerami R, et al. False negative results in Bartonellosis diagnosis. Vector Borne Zoonotic Dis. 2019;19:453-4.

15. Portillo A, Maggi R, Oteo JA, Bradley J, García-Álvarez L, SanMartín M, et al. Bartonella spp. prevalence (serology, culture, and PCR) in sanitary workers in La Rioja Spain. Pathogens. 2020;9:189.

16. Vermeulen MJ, Herremans M, Verbakel H, Bergmans AM, Roord JJ, van Dijken PJ, et al. Serological testing for Bartonella henselae infections in The Netherlands: clinical evaluation of immunofluorescence assay and ELISA. Clin Microbiol Infect. 2007;13:627-34.

17. Penn EB Jr, Goudy SL. Pediatric inflammatory adenopathy. Otolaryngol Clin North Am. 2015;48:137-51.

18. Jacobs RF, Schutze GE. Bartonella henselae as a cause of prolonged fever and fever of unknown origin in children. Clin Infect Dis. 1998;26:80-4.

19. Hansmann Y, DeMartino S, Piémont Y, Meyer N, Mariet P, Heller $\mathrm{R}$, et al. Diagnosis of cat scratch disease with detection of Bartonella henselae by PCR: a study of patients with lymph node enlargement. J Clin Microbiol. 2005;43:3800-6.

20. Spach DH. Endocarditis caused by Bartonella. [cited 2022 Jan 20]. Available from: https://www.uptodate.com/contents/ endocarditis-caused-by-bartonella 